\title{
The flow of an Oldroyd fluid around a sharp corner
}

\section{E.J. Hinch}

Department of Applied Mathematics and Theoretical Physics, Cambridge University, Silver Street, Cambridge CB3 9EW (UK)

(Received March 22, 1993; in revised form May 28, 1993)

\begin{abstract}
A similarity solution is constructed for the flow of an Oldroyd-B fluid around a $270^{\circ}$ re-entrant corner. The velocity is found to vanish like $r^{5 / 9}$ and the stress to be singular like $r^{-2 / 3}$. A simple cxpression is found for the streamfunction.
\end{abstract}

Keywords: Oldroyd-B fluid; re-entrant corner; similarity solution; stress singularity

\section{Introduction}

When a fluid flows around a sharp corner there is a singularity in the stress. This singularity is an embarrassment in numerical calculations. Some numerical schemes for some elastic liquids fail at smaller and smaller Deborah numbers as the numerical resolution is increased [1]. From an analytic solution for the flow in the neighbourhood of the corner, one would hope to produce a singular numerical element in order to avoid this difficulty. The stress singularity is also a test of the rheology: it is not clear that all constitutive equations behave properly at the very high stresses.

We seek a similarity solution for the flow with a streamfunction $\psi$ of the form

$\psi=Q r^{1+\alpha} f(\theta)$,

where $Q$ is an amplitude determined by conditions away from the corner and $r$ and $\theta$ are polar coordinates centred on the corner. In this flow the velocity behaves like $r^{\alpha}$ near to the corner. Very close to the corner, one hopes that length scales other than the distance to the corner become unimportant and so the flow must take the form of a scaleless power law. While one can hope, there is no certainty that such a flow exists. Moreover 
even if such a steady similarity solution does exist, the fluid may not realise this flow in practice.

Similarity solutions do exist and are realised for some fluids. For a Newtonian fluid, Dean and Montagnon [2] found a simple analytic solution with $\alpha=0.5445$ for a $270^{\circ}$ re-entrant corner. In this solution the stress behaves like $r^{-0.4555}$ near to the corner. For power law fluids with a viscosity $\mu=\gamma^{n-1}$ and for a $270^{\circ}$ corner, Henricksen and Hassager [3] found $\alpha=0.37$ for the thinning $n=0.5$ (stress $r^{-0.31}$ ), and $\alpha=0.640$ for the thickening $n=1.5$ (stress $\left.r^{-0.540}\right)$. For a suspension of rigid rods aligned with the flow, Keiller and Hinch [4] found $\alpha=0.58$ (stress $r^{-0.42}$ ) at an effective concentration of the rods $\phi=5$, and $\alpha=0.62$ (stress $r^{-0.38}$ ) for $\phi=20$. The suspension of rigid rods was able to produce stagnating streamlines $(f=0$ not on the walls) although there were problems in interpreting them in terms of lip vortices. All the above cases for which a similarity solution has been constructed are inelastic fluids without memory, i.e. the stress is determined by the instantaneous local velocity gradient. Non-linear elastic liquids with a memory are more difficult. Evidence for the existence of a similarity solution for an Upper-Convected Maxwell fluid was found by Libscomb et al. [5] and Coates et al. [6] in the numerical solution of the $4: 1$ contraction with a stress singularity between $r^{-0.90}$ and $r^{-10}$ at a Deborah number of 0.65 .

The problem with fluids with memory is that they remember other parts of the flow. We shall thus find that the stress near to the corner is partly determined by the shear environment upstream some way from the corner. Previous attempts [7] using a local analysis may not have fully recognised this hyperbolic nature of the stress equation. We shall find it necessary to consider the evolution of the stress moving along a streamline. We start, in Section 2, upstream where the flow is approximately parallel to the wall, if we assume that there is no lip vortex. Now such a unidirectional flow is a viscometric flow, and so the stress field is well understood there.

The governing equations are the conservation of mass and of momentum $\nabla \cdot u=0$, $\nabla \cdot \sigma=0$,

where inertia is neglected. The constitutive equation for the Oldroyd-B fluid will be taken in the form of a pair of equations

$\boldsymbol{\sigma}=-p \boldsymbol{I}+2 \mu \boldsymbol{E}+G \boldsymbol{A}$,

$\frac{\partial A}{\partial t}+\boldsymbol{u} \cdot \nabla \boldsymbol{A}=\boldsymbol{A} \cdot \nabla \boldsymbol{u}+\nabla \boldsymbol{u}^{\mathrm{T}} \cdot \boldsymbol{A}-\frac{1}{\tau}(A-I)$.

I prefer to express the stress as a Newtonian solvent stress with viscosity $\mu$ plus an elastic polymer contribution with elastic modulus $G$ and polymer 
stretch $A$. According to the second microstructural equation, the polymer stretch seen moving with the fluid is a result of deforming affinely with the fluid (first two terms on the right-hand side) and simultaneously relaxing on a time scale $\tau$.

Now it is possible, and has been common, to eliminate $\boldsymbol{A}$ to form a single equation for the deviatoric part of the stress $\sigma^{\prime}$

$\frac{\delta \boldsymbol{\sigma}^{\prime}}{\delta t}+\frac{1}{\tau} \boldsymbol{\sigma}^{\prime}=2 \mu\left[\frac{\delta \mathbf{E}}{\delta t}+\left(\frac{1}{\tau}+\frac{G}{\mu}\right) \boldsymbol{E}\right]$

where $\delta / \delta t$ is the co-deformational or Oldroyd upper-convected time derivative. Now it has been argued from this equation that near the corner where the velocity gradients are large the two Oldroyd derivatives must balance and so the stress is Newtonian, in fact the solvent stress. What has been overlooked in this argument is the possibility that the two Oldroyd derivatives are the dominant terms of the two sides of the equation yet they do not balance, and so at leading order $\delta \sigma^{\prime} / \delta t=0$. This alternative conclusion is more obvious from the non-eliminated form. The microstructural equation suggests that in regions of weak flow the relaxation dominates with $\boldsymbol{A} \approx \boldsymbol{I}$ and that in regions of strong flow the affine deformation dominates with $\delta A / \delta t \approx 0$. There is of course a question still of whether the elastic stress $G A$ or the solvent stress $2 \mu E$ dominates. We shall find in Section 3 that it is the elastic stress which dominates near the sharp corner.

It is convenient to non-dimensionalise the problem, scaling time with the relaxation time $\tau$, length with $(Q \tau)^{1 / 1-\alpha}$, and stress with the elastic modulus $G$. It is then possible to set $Q=G=\tau=1$ in the above equations.

\section{The upstream viscometric region far from the corner and near to the walls}

To calculate the stress for fluids with memory it is necessary to go upstream to where the flow is weak and the microstructure little deformed. Upstream from the corner all the geometrically self-similar streamlines become tangential to the wall, so long as we assume that there is no lip vortex. The no-slip boundary condition means that the flow here is predominantly simple shear. Moreover, because the fluid near to the wall is moving slowly it sees a quasi-steady shear.

Now on the upstream wall, which we shall take to be the direction $\theta=0$, both components of the velocity vanish. With a streamfunction $\psi=r^{1+x} f(\theta)$, this no-slip boundary condition gives $f(0)=f^{\prime}(0)=0$. It is tempting therefore to assume that near to the wall $f \approx-\theta^{2}$, the minus sign indicating that the flow is towards the corner. Unfortunately as we shall find later this quadratic form does not satisfy the momentum equation. Instead we need a different power $f \approx-\theta^{n}$, with $n>1$ in order to satisfy the 
boundary conditions. When the whole coupled problem is resolved, we shall find $n=7 / 3$.

In the upstream viscometric region, we therefore take the streamfunction to be of the form

$\psi=-r^{1+\alpha} \theta^{n}$.

This then gives a velocity

$u_{r}=\frac{1}{r} \frac{\partial \psi}{\partial \theta}=-n r^{\alpha} \theta^{n-1}$

and

$u_{\theta}=-\frac{\partial \psi}{\partial r}=-(1+\alpha) r^{\alpha} \theta^{n}$.

Because $\theta \ll 1$, the radial flow dominates the flow in the angular direction, i.e. at a distance from the corner the flow is unidirectional. Two components of the velocity gradient are important, the shear-rate $\gamma$ and the radial strain-rate $e$

$\gamma=\frac{1}{r} \frac{\partial u_{r}}{\partial \theta}=-n(n-1) r^{\alpha-1} \theta^{n-2}$

and

$e=\frac{\partial u_{r}}{\partial r}=-n \alpha r^{\alpha-1} \theta^{n-1}$.

Again because $\theta \ll 1$ the simple shearing motion at $\gamma$ dominates the radial straining.

For the simple shear flow, the polymer stretch is well known to be $A_{\theta \theta}=1, \quad A_{\theta r}=\gamma$ (shear stress), $\quad A_{r r}=1+2 \gamma^{2}$ (normal stress).

When the flow is very weak with $\gamma \ll 1$ far upstream, the polymer is fully relaxed and $\boldsymbol{A} \approx \boldsymbol{I}$. As the fluid moves towards the corner, a Newtonian shear stress develops in $A_{\theta r}$. Nearer the corner, once the local Deborah number equals unity, i.e. $\gamma=1$ in the non-dimensionlisation, the normal stress grows to dominate the stress.

Following along a streamline towards the corner, the viscometric region breaks down when the radial stretching becomes important, i.e. $e=O(1)$. At the same time the quasi-steady approximation is lost as the rate of change of the shear-rate seen moving with the fluid becomes important, i.e. $\boldsymbol{u} \cdot \nabla=O(1)$. For streamlines which go sufficiently close to the corner, this breakdown occurs near to the wall, $\theta \ll 1$. Thus at the breakdown the flow is still mainly radial, and the shearing $\gamma$ is much larger than the radial 
stretching $e$ so that the polymer stretch $A$ is dominated by the normal stress term $A_{r r}$.

After the breakdown of the viscometric approximation, the fluid moves rapidly around the corner in a small region of high velocity gradients. In the polymer stretch equation, the advection and affine deformation terms dominate and we can neglect the relaxation term. Thus the polymer will deform affinely with the fluid. Since on entering this region the polymer stress is dominated by the streamwise normal stress term, within the region the polymer stretch must be proportional to the square of the velocity

$A=g(\psi) u \boldsymbol{u}$,

where the constant of proportionality $g$ can be different on different streamlines. We determine $g(\psi)$ shortly.

This behaviour, dominated by relaxation until the strain-rate exceeds the relaxation rate (appropriate Deborah number exceeds unity) and thereafter showing affine deformation, is seen from a Lagrangian perspective in similar rapidly varying flows, e.g. in sink flow [8], in fibre spinning with a sufficiently low initial strain-rate and the related stretching of a uniform column by a fixed force [9]. The complication in the flow around a sharp corner is that the transition to affine deformation occurs only when the radial straining becomes large, not at the earlier time when the shear-rate becomes large. The behaviour was however noticed in flow around a sharp corner by Keiller [10] and Renardy [11].

To confirm the behaviour suggested above, the polymer stretch equation was solved numerically in polar coordinates in the near wall region where $\psi=-r^{1+\alpha} \theta^{n}$. The governing equations are

$\frac{\mathrm{d} A_{r r}}{\mathrm{~d} r}=\frac{2 \alpha}{r} A_{r r}+\frac{2(n-1)}{r \theta} A_{r \theta}+\frac{1}{n r^{\alpha} \theta^{n-1}}\left(A_{r r}-1\right)$,

$\frac{\mathrm{d} A_{r \theta}}{\mathrm{d} r}=\frac{\left(1-\alpha^{2}\right) \theta}{n r} A_{r r}+\frac{(n-1)}{r \theta} A_{\theta \theta}+\frac{1}{n r^{\alpha} \theta^{n-1}} A_{r \theta}$,

$\frac{\mathrm{d} A_{\theta \theta}}{\mathrm{d} r}=\frac{2\left(1-\alpha^{2}\right) \theta}{n r} A_{r \theta}-\frac{2 \alpha}{r} A_{\theta \theta}+\frac{1}{n r^{\alpha} \theta^{n-1}}\left(A_{\theta \theta}-1\right)$.

These equations have been solved numerically along a streamline $\psi=-c$ where $\theta=c^{1 / n} r^{-(1+\alpha) / n}$, starting from large $r$ where $A \approx I$ and integrating towards the corner $r=0$.

Figure 1 shows the radial dependence of the polymer stretch $A_{r r}$ along the streamline $\psi=-10^{-3}$. In $r>3$ the polymer stretch is relaxed with $A_{r r} \approx 1$. The shear-rate $\gamma(r)$ becomes equal to unity at $r=1.25$ on this streamline. In the range $0.5<r<3$, the polymer stretch is that in simple shear with $A_{r r} \approx 1+2 \gamma^{2}$. The radial strain-rate $e$ becomes equal to unity at $r=0.063$ 


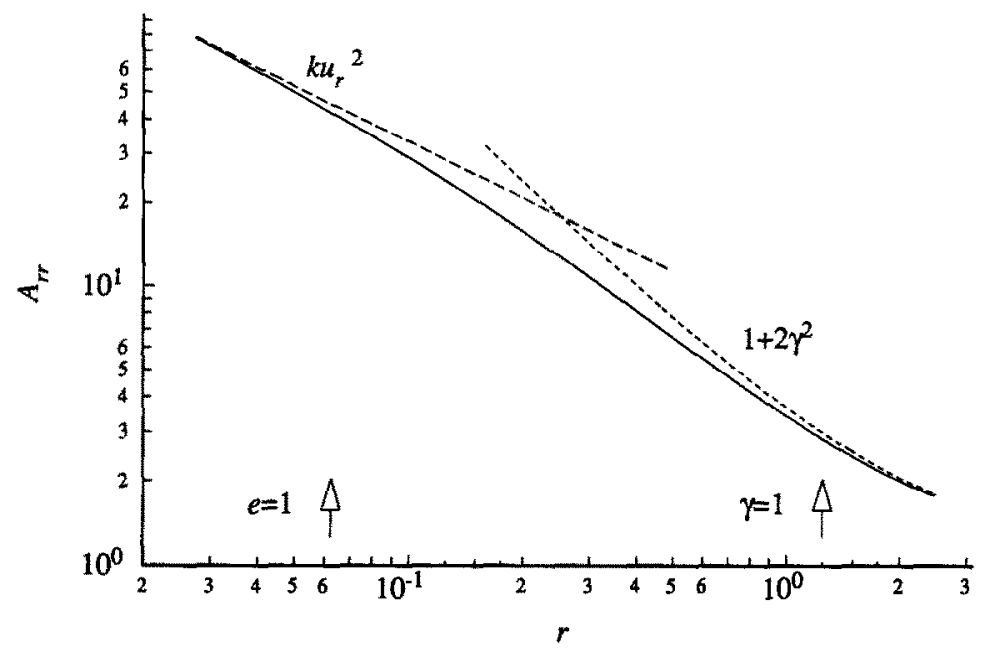

Fig. 1. The variation of the polymer stretch $A_{r r}$ with radial position $r$ along the streamline $\psi=-10^{-3}$ in the viscometric region.

on the streamline. For $r<0.1$ the polymer stretch follows the affine deformation $A_{r r} \approx k u_{r}^{2}$, with $k=3.51 \times 10^{3}$. The same behaviour is found on other, geometrically similar, streamlines. Note that on the streamline $\psi=-10^{-3}$ the radial strain-rate $e=1$ when $\theta=0.34$, which is not small compared with unity, and this angle only decreases to 0.2 on $\psi=-10^{-5}$.

To find the important variation of the polymer stretch across the streamlines as described by $g(\psi)$, we need to examine the scaling of the stress in the viscometric region where $\psi \approx-r^{1+\alpha} \theta^{n}$. On the streamline $\psi=-c$, the angle to the corner $\theta$ is related to the radial distance by $\theta=c^{1 / n} r^{-(1+\alpha) / n}$.

Using this to eliminate $\theta$, the expressions above for the radial velocity, shear-rate and radial strain-rate have radial dependencies along the streamline given by

$$
\begin{aligned}
& u_{r}=-n c^{(n-1) / n} r^{-(n-1-\alpha) / n}, \\
& \gamma=-n(n-1) c^{(n-2) / n} r^{-2(n-1-\alpha) / n}
\end{aligned}
$$

and

$e=-n \alpha c^{(n-1) / n} r^{-(2 n-1-\alpha) / n}$.

The viscometric approximation breaks down when $e=O(1)$, i.e. when $r=O\left(c^{(n-1) /(2 n-1-\alpha)}\right)$. 
At this position we therefore have the estimates for the polymer stretch and the radial flow

$$
A_{r r}=O\left(\gamma^{2}\right)=O\left(c^{-2(1-\alpha) /(2 n-1-\alpha)}\right)
$$

and

$u_{r}=O\left(c^{(n-1) /(2 n-1-\alpha)}\right)$.

Thus at the breakdown we have

$A_{r r}=g(\psi) u_{r}^{2}$,

with

$g(-c)=1.31 c^{-\beta}, \quad \beta=\frac{2(n-\alpha)}{2 n-1-\alpha}$.

The numerical factor 1.31 is determined from the numerical solution of the full equations near the wall.

We have now determined the stress distribution as it leaves the upstream viscometric region near the wall in terms of the unknown parameters $\alpha$ and $n$.

\section{The core region near the corner and far from the walls}

Near the corner there are high velocity gradients, so that the relaxation of the polymer is negligible and instead the polymer deforms affinely with the fluid. Note that although the velocity tends to zero as $r \rightarrow 0$ it does so with infinite gradient and so the residence time of the polymer in the corner region tends to zero while the fluid deformation tends to infinity. We assume that the elastic stress from the deformed polymer dominates the Newtonian solvent stress near to the corner, an assumption which must be checked when we have completed the solution. Thus near the corner we have

$$
\boldsymbol{\sigma}=-p \boldsymbol{I}+g(\psi) \boldsymbol{u} \boldsymbol{u} \text {. }
$$

This form of the stress is rather like the Reynolds stress in inviscid fluid mechanics, with a 'density' $g$ strongly varying across the streamlines.

Substituting this stress into the momentum equation (with no inertia), we obtain

$0=-\nabla p+g u \cdot \nabla u$,

where we used the incompressibility condition $\nabla \cdot u=0$ and the kinematic requirement that along a streamline $\boldsymbol{u} \cdot \nabla \boldsymbol{g}=0$. The pressure can be elimi- 
nated by taking the curl of the momentum equation, so forming the vorticity equation

$0=g \boldsymbol{u} \cdot \nabla \omega-\frac{\mathrm{d} g}{\mathrm{~d} \psi} \boldsymbol{u} \cdot \nabla \frac{1}{2} u^{2}$,

where $\omega$ is the vorticity $\nabla \wedge \boldsymbol{u}$. It is now time to substitute the similarity form of the streamfunction $\psi=-r^{1+\alpha} f(\theta)$ and the result from the previous section for the variation of the stress across the streamlines $g \propto \psi^{-\beta}$. We thereby obtain the equation governing the similarity profile

$f^{\prime \prime \prime}=a_{1} \frac{f^{\prime} f^{\prime \prime}}{f}+a_{2} f^{\prime}+a_{3} \frac{f^{\prime 3}}{f^{2}}$

where the coefficients are given by

$a_{1}=\beta-\frac{1-\alpha}{1+\alpha}, \quad a_{2}=(\beta-2)(1+\alpha), \quad a_{3}=-\frac{\alpha \beta}{1+\alpha}$.

This equation must be solved with the no-slip boundary conditions $f(0)=f^{\prime}(0)=f\left({ }_{2}^{3} \pi\right)=f^{\prime}\left(\frac{3}{2} \pi\right)=0$.

This constitutes a non-linear eigenvalue problem for the unknown $\alpha$ and $n$ (and hence $\beta$ ).

The governing equation is quasi-linear, in that if $f$ is a solution then any multiple of $f$ is also a solution. Thus the similarity solution has the same pattern of streamlines as the strength of the flow changes, a feature shared with similarity solutions for other fluids. A more unusual feature is that the stress (scaled on $G$ ) is predicted to be independent of the strength of the flow at a fixed non-dimensional distance from the corner (scaled on $\left.\left(Q_{\tau}\right)^{1 /(1-\alpha)}\right)$.

Another surprising feature of the governing differential equation is that it is of third and not fourth order. This is a result of the stress involving only the velocity and not the velocity gradient. Now one solution of the non-linear equation is $f^{\prime} \equiv 0$ corresponding to circular streamlines with a constant tangential velocity. This feature of the third-order equation, that $f^{\prime \prime \prime}=0$ when $f^{\prime}=0$, means that the flow is symmetric about the directions where $f^{\prime}=0$; a symmetry in which $f$ (i.e. $u_{\theta}$ ) and $f^{\prime \prime}$ are even and $f^{\prime}$ (i.e. $u_{r}$ ) is odd. Thus to find the flow around a sharp corner from $\theta=0$ to $\theta=3 \pi / 2$, we need only consider the half problem from the upstream wall $\theta=0$ to the symmetry direction $\theta=3 \pi / 4$. The symmetry means that the core region near the corner will deliver the polymer to the downstream wall region in the same state as it left the upstream wall region, and so the downstream wall region will be a symmetric version of the upstream wall region with no new large stresses there. 
The governing third-order equation is singular as $f \rightarrow 0$. An initial assumption that $f \approx-\theta^{2}$ leads to $f^{\prime \prime \prime} \propto \theta^{-1}$ which is inconsistent. If we try instead $f \approx-\theta^{n}$ then we find that it is possible to balance three of the four terms in the equation if

$n(n-1)(n-2)=a_{1} n^{2}(n-1)+a_{3} n^{3}$.

Substituting in the expressions for the coefficients $a_{1}$ and $a_{3}$, we find $\beta=2(n-1) / n$,

independent of the value of $\alpha$. This result implies that as one approaches the wall from the core region the stress $g u_{r} u_{r}$ is independent of $\theta$ at each $r$. Now the viscometric region investigated in the previous section gave an expression for $\beta$ in terms of $\alpha$ and $n$. Eliminating $\beta$ we find

$\alpha=3 n-n^{2}-1$

Now the non-linear third-order differential equation for $f(\theta)$ does have a simple closed-form analytic solution. Some preliminary numerical solutions revealed a simple sinusoidal structure for $f(\theta)$. There is an obvious exact solution of simple shear $\psi=r^{2} \sin ^{2} \theta$ for a plane boundary with no sharp corner, for which the stress is constant. These hints together with the singular behaviour discussed in the previous paragraph suggest seeking a solution in the form

$f=-m^{-n} \sin ^{n} m \theta$.

Substituting this into the governing equation we find

$$
\begin{aligned}
n(n-1)(n-2)-n^{3} \sin ^{2} m \theta= & a_{1}\left[n^{2}(n-1)-n^{3} \sin ^{2} m \theta\right] \\
& +a_{2} m^{-2} n \sin ^{2} m \theta+a_{3} n^{3}\left(1-\sin ^{2} m \theta\right) .
\end{aligned}
$$

The terms independent of $\theta$ give the same balance as in the analysis of the singularity as $f \rightarrow 0$, and so will cancel with the same choice of $\beta$. The terms proportional to $\sin ^{2} m \theta$ givc, after substituting the expressions for the $a_{t}$,

$m=3-n$.

The problem is finally resolved by selecting $m=2 / 3$ appropriate to the $270^{\circ}$ corner, so that the streamfunction vanishes at the two walls $\theta=0$ and $\theta=3 \pi / 2$. Note that the above solution for $f$ is applicable to all sharp re-entrant corners, from a plane boundary $\left(180^{\circ}\right.$ corner $)$ with $m=1$ to complete $360^{\circ}$ turn with $m=1 / 2$. For the $270^{\circ}$ corner, we need $m=2 / 3$ and so we find

$n=\frac{7}{3}, \quad \alpha=\frac{5}{9}, \quad \beta=\frac{8}{7}, \quad \psi=-\left(\frac{3}{2}\right)^{7 / 3} r^{14 / 9} \sin ^{7 / 32} \frac{2}{3} \theta$. 
Now in the core region the elastic stress has the form $1.31(-\psi)^{-\beta} u u$. Substituting our solution for the streamfunction, we find a stress

$$
\begin{aligned}
& \sigma_{r r}=-p+K r^{-2(n-2)}(3-n)^{2} n^{2} \cos ^{2} m \theta, \\
& \sigma_{r \theta}=-K r^{-2(n-2)}(3-n) n\left(3 n-n^{2}-1\right) \sin m \theta \cos m \theta, \\
& \sigma_{\theta \theta}=-p+K r^{-2(n-2)}\left(3 n-n^{2}-1\right)^{2} \sin ^{2} m \theta,
\end{aligned}
$$

where $K=1.31 m^{-6 / n}$. Thus for the $270^{\circ}$ corner the elastic stress has a singularity with a radial dependence $r^{-2 / 3}$ in the core region near to the corner away from the walls. The solvent stress has a weaker singularity like $r^{-4 / 9}$ and hence it was correct to neglect it in the analysis. (For a general corner, the solvent stress has a singularity like $r^{-1+\alpha}=r^{-2+3 n-n^{2}}$ which is less singular than the elastic stress like $r^{-2(n-1)}$ while $1<n<4$, which it is for all re-entrant corners.) The stress in the viscometric region has a singularity like $r^{-4 / 9}$ in $\sigma_{r \theta}$ and like $r^{-8 / 9}$ in $\sigma_{r r}$, these variations being for fixed $\theta$ rather than fixed $\psi$.

\section{Conclusions}

A similarity solution has been found for the Oldroyd-B fluid flowing around a sharp corner, with a velocity varying like $r^{5 / 9}$ and a stress singularity like $r^{-2 / 3}$. This behaviour has not yet been seen in numerical solutions [5,6], although the reported stresses between $r^{-0.9}$ and $r^{-1.0}$ may be the $r^{-8 / 9}$ in the viscometric region near the walls. It may be that the numerical methods were not working well near the corner, or that the similarity region is too small to resolve. There are certainly difficulties integrating the stress equation near to the no-slip wall. On the other hand, the above similarity solution may be unstable or there may be other possible solutions, perhaps not of a similarity type.

The behaviour near the corner is controlled by the distribution of stress across the streamlines, $g(\psi)$, which is determined some way upstream from the corner. In this paper it was assumed that the similarity velocity field was applicable there, at least as far upstream as where the Deborah number becomes less than unity. Future studies should generalise this assumption. Thus lip vortices have been excluded by assuming that upstream the fluid comes from near to the walls. A preliminary investigation suggests that lip vortices would radically change the above analysis, by making $g(\psi)$ constant and so making the elastic stress smaller than the solvent stress. To test the theory of this paper, perhaps some full numerical solutions could be made in geometries which inhibited a lip vortex, e.g. flow around an abrupt junction in a channel.

In the corner region it was found that the $r^{-2 / 3}$ elastic stress dominated the $r^{-4 / 9}$ solvent stress, so that in a sense the analysis is for a Maxwell fluid. 
In fact the calculation does not depend on the value of the non-dimensional group $G \tau / \mu$ so long as it is not zero. Thus the analysis is applicable to all Oldroyd fluids other than the Newtonian fluid with $G \tau / \mu=0$.

Finally, it should be pointed out that the analysis has neglected the polymer relaxation in the core region and has not satisfied the momentum equation very close to the walls in the viscometric region. There is certainly a possibility that these small effects might destroy the above solution.

\section{References}

1 R. Keunings, J. Non-Newtonian Fluid Mech., 20 (1986) 209-226.

2 W.R. Dean and P.E. Montagnon, Trans. R. Soc. London Ser. A., 308 (1949) 199-218.

3 P. Henricksen and O. Hassager, J. Rheol., 33 (1989) 865-879.

4 R.A. Keiller and E.J. Hinch, J. Non-Newtonian Fluid Mech., 40 (1992) 323-335.

5 G.G Libscomb, R. Keunings and M.M. Denn, J. Non-Newtonian Fluid Mech., 24 (1987) $85-96$.

6 P.J. Coates, R.C. Armstrong and R.A. Brown, J. Non-Newtonian Fluid Mech., 42 (1992) $141-188$.

7 A.R. Davies, J. Non-Newtonian Fluid Mech., 29 (1988) 269-293.

8 J.M. Rallison and E.J. Hinch, J. Non-Newtonian Fluid Mech., 29 (1988) 37-55

9 E.J. Hinch, in Y. Rabin (Ed.), Polymer-Flow Interactions, American Institute of Physics, 1985 , pp. $59-69$.

10 R.A. Keiller, Ph.D. Thesis, Cambridge University, 1991, pp. 165-169.

11 M. Renardy, J. Non-Newtonian Fluid Mech., 50 (1993) 127-134. 